[8] M. Born and E. Wolf, Principle of Optics. New York: Pergamon, 1965 , ch. 10.

[9] R. J. Collier, C. B. Burckhardt, and L. H. Lin, Optical Holography. New York: Academic, 1971, ch. 7.

[10] I. H. Malitson, "Interspecimen comparison of the refractive index of fused silica," J. Opt. Soc. Amer., vol. 55, no. 10, pp. 12051209,1965 .

[11] M. J. Adams, D. N. Payne, F. M. E. Sladen, and A. H. Hartog, "Wavelength-dispersion properties of glasses for optical fibers: The germania enigma," Electron. Lett., vol. 14, no. 22, pp. 703-705, 1978.

[12] F. M. E. Sladen, D. N. Payne, and M. J. Adams, "Definitive profile-dispersion data for germania-doped silica fibers over an extended wavelength range," Electron. Lett., vol. 15, no. 15, pp. 469-470, 1979 .

[13] D. Gloge, "Weakly guiding fibers," Appl. Opt., vol. 10, no. 10, pp. 2252-2258, 1971.

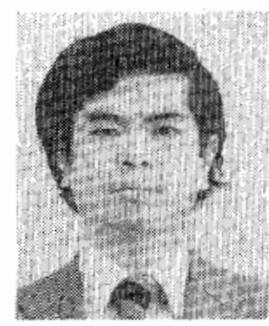

Mitsuhiro Tateda was born in Kochi, Japan, on September 1, 1949. He received the B.Sc., and M.Sc. degrees in physical engineering from the University of Tokyo, Tokyo, Japan, in 1972 and 1974 , respectively.

From 1974 to 1977 he was a member at Musashino Electrical Communication Laboratory, Nippon Telegraph and Telephone Public Corporation, Musashino, Tokyo, Japan. Since 1977 he has been an Engineer at Ibaraki Electrical Communication Laboratory, N.T.T.,
Tokai, Ibaraki, Japan. He has been working on optical fiber transmission systems. His main interest is in investigating the transmission characteristics of optical fibers, multimode graded-index and singlemode fibers, for the practical design of trunc-line optical cables.

Mr. Tateda is a member of the Optical Society of America and the Institute of Electronics and Communication Engineers of Japan.

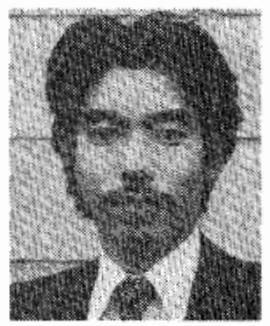

Nori Shibata was born in Kanagawa Prefecture, Japan, on September 30, 1952. He received the B.E. degree in physical engineering, and the M.E. degree in information processing, both from the Tokyo Institute of Technology, Tokyo, Japan, in 1976 and 1978, respectively.

In 1978 he joined the Ibaraki Electrical Communication Laboratory, Nippon Telegraph and Telephone Public Corporation, Tokai, Ibaraki, Japan, where he has been engaged in research work on transmission characteristics of optical fiber cables. His current interests are in graded-index and singlemode fibers.

Mr. Shibata is a member of the Institute of Electronics and Communication Engineers of Japan.

Shigeyuki Seikai, for a photograph and biography, see page 39 of the January 1981 issue of this JoURNAL.

\title{
Planar Multimode Detector Arrays for Infrared and Millimeter-Wave Applications
}

\author{
D. B. RUTLEDGE, MEMBER, IEEE, AND S. E. SCHWARZ
}

\begin{abstract}
A new type of detector array is described. By means of a suitably designed metallic network, many detector elements (each individually small compared to wavelength) are assembled into an impedancematched termination for radiation incident normally on the plane of the device. Residual reactance is tuned out by means of a movable backshort. An array of $\mathbf{4 0 0}$ bismuth-film microbolometers with a total area of $1 \mathrm{~cm}^{2}$ has been tested at $215 \mathrm{GHz}$. A coupling efficiency of approximately 60 percent was observed. The detector has a $D^{*}$ of $4 \times 10^{8} \mathrm{~cm} \cdot \mathrm{Hz}^{1 / 2} / \mathrm{W}$ at room temperature with response time on the order of $2 \times 10^{-7} \mathrm{~s}$. Similar arrays of Schottky and SIS diodes can probably be constructed.
\end{abstract}

Manuscript received June 6, 1980; revised September 23, 1980. This work was supported by the National Science Foundation under Grant ENG78-13933, by the U.S. Army Research Office under Grant DAAG2979-C-0134, and by the Joint Services Electronics Program under Contract F49620-79-C-0178.

D. B. Rutledge was with the Department of Electrical Engineering and Computer Sciences and the Electronics Research Laboratory, University of California, Berkeley, CA 94720 . He is now with the Department of Electrical Engineering, California Institute of Technology, Pasadena, CA 91125.

S. E. Schwarz is with the Department of Electrical Engineering and Computer Sciences and the Electronics Research Laboratory, University of California, Berkeley, CA 94720.

\section{INTRODUCTION}

$\mathrm{D}$ EPENDING on the nature of the radiation being received, there are occasions when a collection of antennas will provide better performance than a single receiving antenna. This is not the case when the radiation originates from a point source or its equivalent [Fig. 1(a)]. With a point source, the received power $P$ is given by

$$
P=S A
$$

where $S$ is the power density at the antenna and $A$ is the effective area of the antenna. In principle, the received power can be made as large as necessary by increasing the effective area, and hence there is no need for more than one antenna. On the other hand, the source may be distributed [Fig. 1(b)] as in plasma studies, cosmic-background measurements, or terrestrial radiometric mapping. Distributed sources are usually characterized by an effective brightness temperature $T$. If the source temperature is constant over the beamwidth of a single antenna, and if the antenna is matched and lossless, the received power per unit bandwidth is given by Nyquist's 

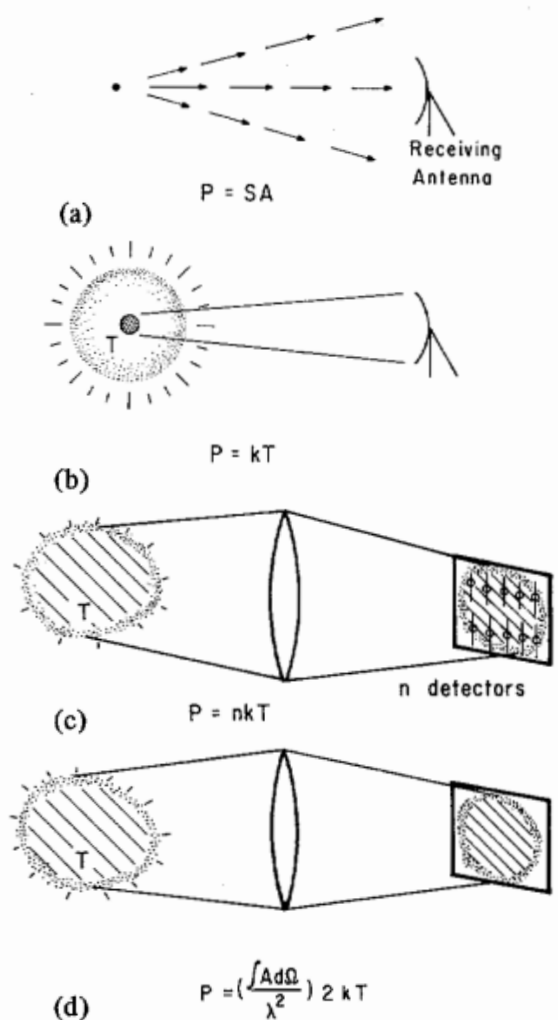

Fig. 1. (a) Single-mode antenna receiving from a point source. (b) Single-mode antenna receiving from a distributed source. (c) Multimode antenna receiving from a distributed source. (d) Large-area detector receiving from a distributed source.

theorem [1], valid for $h \nu \ll k T$ :

$$
P=k T \text {. }
$$

This is just the same as the power available from a single-mode waveguide terminated in its characteristic impedance. In this case one cannot increase the received power by increasing the effective area because the beamwidth narrows at the same time. However, we can increase the received power by adding more antennas, each with its own matched load, as indicated in Fig. 1(c). Here a lens is used to focus an image of the source on a collection of $n$ antennas. Assuming that the antennas do not interact, the received power per unit bandwidth will now be given by

$$
P=n k T \text {. }
$$

This is the same as the power available from a terminated waveguide supporting $n$ nodes, and hence we shall refer to the collection of antennas, each with its own load, as an $n$-mode array. We note that it differs from a phased array, which is a single-mode antenna. The pattern of the multimode array is the same as the pattern of one of its elements. This means that the effective area no longer obeys the single-mode antenna theorem [2]

$$
\int A(\Omega) d \Omega=\lambda^{2}
$$

but rather satisfies

$$
\int A(\Omega) d \Omega=n \lambda^{2}
$$

where $n$ is the number of antennas. Conventional large-area infrared detectors [Fig. 1(d)] are similar in this regard. The effective area of a large bolometer or photoconductor does not obey (4), but it can be shown to obey a formula similar to (5), provided that the mode number is suitably defined. It seems reasonable to define $m$, the effective number of modes of any detector, to be equal to $P / k T$ where $P$ is the power per unit bandwidth received from a distributed source. $P$ is given by

$$
P=\int B A d \Omega
$$

where $B$ is the brightness $\left(\mathrm{W} \cdot \mathrm{Hz}^{-1} \cdot \mathrm{m}^{-2} \cdot \mathrm{sr}^{-1}\right)$. From the Rayleigh-Jeans law, valid for $h \nu \ll k T$, we have

$$
B=\frac{2 k T}{\lambda^{2}} \text {. }
$$

At higher frequencies, both (3) and (7) must be multiplied by the quantum correction factor $h \nu(k T)^{-1}[\exp (h \nu / k T)-1]^{-1}$. Assuming that $T$ is constant over the angle of acceptance of the optical receiver, the received power is then

$$
P=\frac{2 k T \int A d \Omega}{\lambda^{2}} .
$$

According to our definition of $m$, the effective area of the large-area detector therefore obeys $\int A(\Omega) d \Omega=m \lambda^{2} / 2$, which agrees with (5) except for a factor of 2 . The reason for this factor is that the optical detector accepts both polarizations, while the multimode antenna accepts only one.

Since multimode arrays have properties similar to conventional large-area detectors, they can be used in place of pyroelectric detectors or bolometers. One advantage of doing this is that multimode antennas allow us to construct large-area detectors in which the actual detection is performed by very small devices such as microbolometers [4], Schottky diodes [5], and SIS junctions [6]. This can lead to significant improvements in detectivity and speed. There are also some other potential advantages. Traditional thermal detectors usually have to be shielded from room-temperature thermal radiation. Multimode antennas do not have to be shielded from room-temperature radiation because most of that radiation is in the middle infrared. Antennas designed for the submillimeter range will have very small effective areas at other wavelengths. In effect, a bandpass filter is built into the device. Thus, its specific detectivity will not be limited by the room-temperature background-limited $D^{*}$ of $1.2 \times 10^{11} \mathrm{~cm}$. $\mathrm{Hz}^{1 / 2} / \mathrm{W}$ often ascribed to thermal detectors [7].

Multimode antennas can be constructed by means of planarprocessing techniques. The resulting monolithic structures can thus be expected to be relatively inexpensive and reliable. The size of the repetitive structures may, in some cases, be limited by the practical accuracy of mask registration over large areas; this will depend on the alignment accuracy required by each individual detector. In the less demanding cases, arrays several centimeters in diameter can be built. The general form of a typical multimode array is shown in Fig. 2. It consists of a grating of wide metal sheets connected by narrow metal 


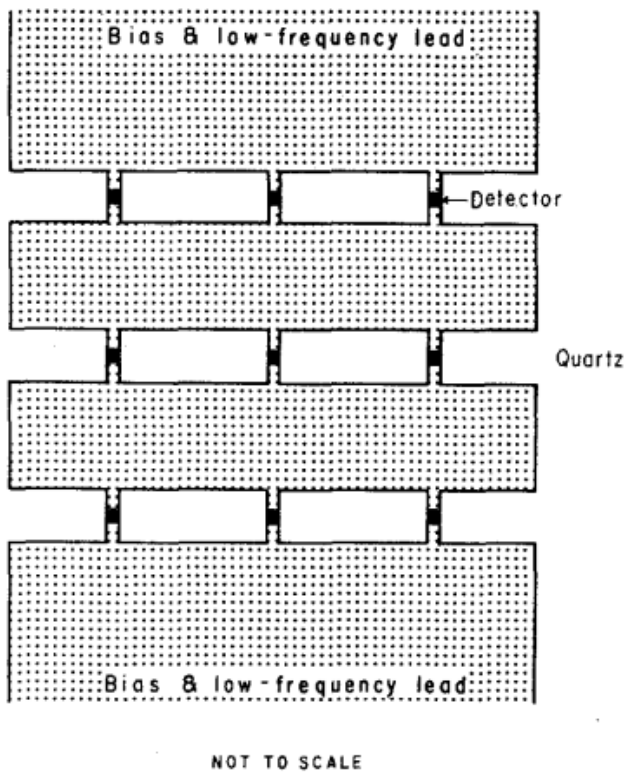

Fig. 2. Schematic design of multimode antenna.

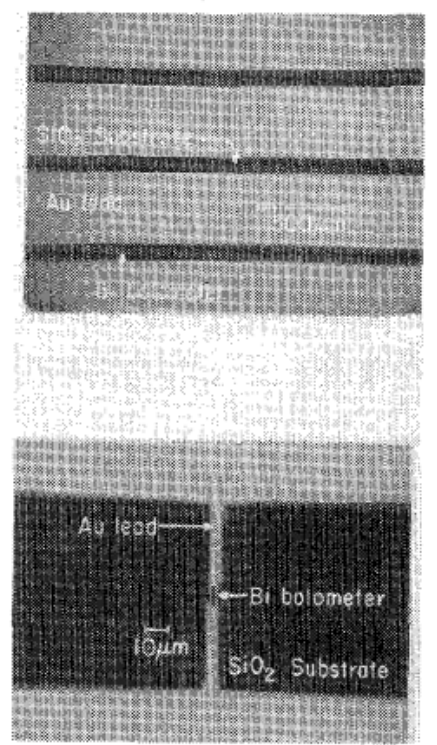

Fig. 3. Photomicrographs of microbolometer array.

strips with detectors on them. We shall refer to the detectorcontaining strips as "leads." Bias and low-frequency connections are made to the top and bottom sheets. The number of detectors shown in this figure has been reduced for the sake of clarity.

In our experiments we have used 400 element arrays of bismuth-film microbolometers, intended for detection in the range $150-300 \mathrm{GHz}$. Each array contains 20 rows of $20 \mathrm{mi}$ crobolometers spaced at intervals of $500 \mu \mathrm{m}$; thus, the total detector area is $1 \mathrm{~cm}^{2}$. A photograph of a typical array is shown in Fig. 3. The substrate, which appears black on the micrograph, is a fused-quartz cover slip about $150 \mu \mathrm{m}$ thick. The metal leads are gold $(100 \mathrm{~nm}$ thick, on top of a $10 \mathrm{~nm}$ layer of nichrome for adhesion). The bolometers are similar to those of [4], with bismuth films 100 nm thick. The gap between the wide metal sheets is $87 \mu \mathrm{m}$, and the narrow leads containing the bolometers are $6 \mu \mathrm{m}$ wide. The bolometers themselves are $3 \mu \mathrm{m}$ square. Both the gold and bismuth pat-

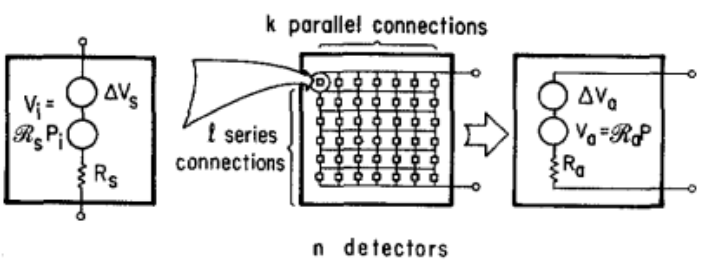

Fig. 4. Thevenin equivalent circuits for the detector array.

terns are defined by lift-off. Next we shall discuss design principles of the device, after which experimental results will be described.

\section{NEP AND DETECTIVITY}

Fig. 4 shows, on the left, the low-frequency equivalent circuit of a single detector, consisting of a source resistance $R_{s}$ in series with an rms noise voltage $\Delta V_{s}$ and a signal voltage $V_{i}$, given by

$$
V_{i}=R_{s} P_{i} .
$$

Here $R_{s}$ is the voltage responsivity of a single device and $P_{i}$ is the power absorbed by the $i$ th device. The devices are connected in a rectangular array with $k$ parallel connections and $l$ series connections as shown in the middle of the figure. An equivalent circuit for the entire array is shown on the right side of the figure. The array's resistance is

$$
R_{a}=R_{s}(l / k) \text {. }
$$

Assuming that the noise voltages are independent, the noise voltage $\Delta V_{a}$ is given by

$$
\Delta V_{a}=\Delta V_{s}(l / k)^{1 / 2}
$$

and the signal voltage $V_{a}$ is

$$
V_{a}=\left(R_{s} / k\right) P
$$

where $P$ is the total power absorbed by the array. Thus, the voltage responsivity of the array $\mathbb{R}_{a}$ is given by

$$
R_{a}=R_{s} / k \text {. }
$$

For a single detector, the noise-equivalent power (NEP) is given by

$$
\mathrm{NEP}_{s}=\frac{\Delta V_{s}}{R_{s}}
$$

and for the array by

$$
\mathrm{NEP}_{a}=\frac{\Delta V_{a}}{R_{a}}=\sqrt{n} \mathrm{NEP}_{s}
$$

where $n$ is the number of devices in the array. Thus, the NEP is proportional to the square root of the number of devices, and assuming that the devices are uniformly distributed, to the square root of the array area. Accordingly, the array can be characterized by a specific detectivity $D^{*}$ given by

$$
D^{*}=\frac{\sqrt{A_{a}}}{\mathrm{NEP}_{a}}=\frac{\sqrt{A_{s}}}{\mathrm{NEP}_{s}}
$$

where $A_{a}$ is the area of the array and $A_{s}$ is the area allotted to each element. If the unit cell of the array is square, then 


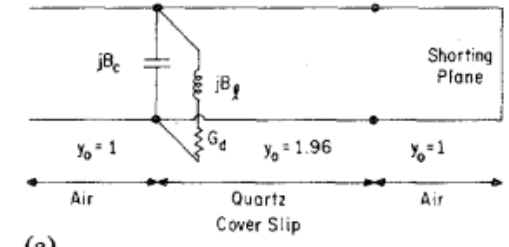

(a)

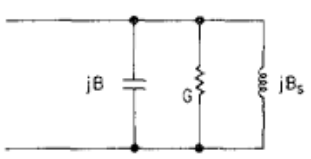

(b)

Fig. 5. (a) Transmission-line equivalent circuit of the array. (b) Simplified equivalent circuit.

$\sqrt{A_{s}}$ is just the period of the array. The above calculation of $D^{*}$ assumes that the efficiency with which incident power is coupled into the detectors is unity. If the actual coupling efficiency is $\eta, D^{*}$ must be decreased by this factor. Expression (16) allows us to estimate the performance of an array of devices from the NEP of a single device. For example, the elements of the microbolometer arrays to be described below are spaced at intervals of $0.05 \mathrm{~cm}$ and each bolometer has an NEP of about $10^{-10} \mathrm{~W} / \mathrm{Hz}^{1 / 2}$. Thus, one expects that the array will have a $D^{*}$ on the order of $5 \times 10^{8} \mathrm{~cm} \cdot \mathrm{Hz} z^{1 / 2} / \mathrm{W}$.

\section{High-FrequenCy Design}

The goal of the array design is that all the incident radiation should be conducted to the detector elements and absorbed by them. Ideally, no radiation should be reflected. Moreover, it is desirable to use as few detectors as possible because the NEP is proportional to $\sqrt{n}$. However, there is an upper limit on the spacing that can exist between rows or columns of the periodic structure. This limit is imposed by the possibility that the structure may act as a grating, diffracting energy into new reflected or transmitted waves or into surface waves. To prevent this, the periods in our design were chosen to be $2 / 3$ of the wavelength in the substrate material. The important dimensions of the array-the gap width and lead width-are chosen by treating the array as though it were infinite in extent and using the equivalent transmission-line circuit shown in Fig. 5. Assuming that the wave is incident normally, we can represent the air above the grating by a transmission line with normalized admittance equal to one. The gap is represented by a normalized capacitive susceptance $B_{c}$. Parallel to this is the inductive susceptance of the leads $B_{l}$ in series with the detector conductance $G_{d}$. The parasitic capacitance parallel to the detector is neglected. The quartz is represented by a section of line having a characteristic admittance equal to its refractive index of 1.96 . Behind the substrate is a polished copper plate that acts as a shorting plane. This short, together with the section of transmission line in front of it, acts as an adjustable susceptance. We wish to choose the gap and the lead width so that the detector acts as a susceptance $B$ in parallel with a conductance $G$ that is nearly equal to unity. The susceptance can then be tuned out by moving the shorting plane.

The values of the circuit parameters can be calculated in two ways. The first is a quasi-static method that is simple, but

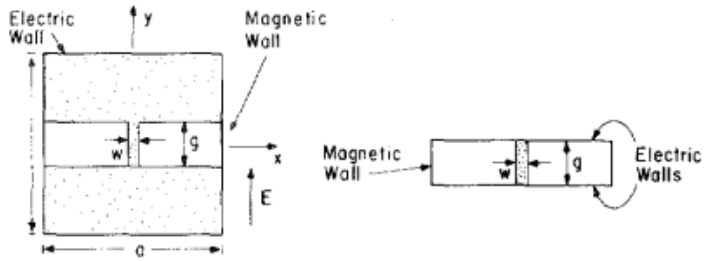

(a)

(b)

Fig. 6. (a) Equivalent square waveguide configuration for circuit anallysis. (b) Waveguide configuration for inductance calculation.

only accurate enough for preliminary calculations. The second is a modification of the EMF method developed and extensively verified in a classic paper by Eisenhart and Khan [8]. It is accurate, but not simple. In both analyses we reduce the array to a single waveguide bounded on the top and bottom by electric walls and on the sides by magnetic walls, as shown in Fig. 6(a). The quasi-static analysis proceeds as follows. The capacitance of the gap is assumed to be unaffected by the presence of the lead, by the shorting plane, or by the fact that the substrate has only a finite thickness. It is then calculated by conformal mapping [9]. The result is

$$
B_{c}=\frac{2 k_{0} a}{\pi}\left(\frac{1+\epsilon_{r}}{2}\right) \ln \csc \left(\frac{\pi g}{2 a}\right)
$$

where $\epsilon_{r}$ is the dielectric constant of the substrate and $k_{0}$ is the propagation constant of free space. The inductance is calculated by assuming it is the same as for the shortened guide shown in Fig. 6(b). Applying Babinet's principle and duality to the capacitive slot [9], we obtain the result

$$
B_{l}=-\left(\frac{k_{0} g}{2 \pi} \ln \csc \frac{\pi w}{2 a}\right)^{-1} \text {. }
$$

Eisenhart and Khan analyzed a gap-post configuration in a metal waveguide. The present waveguide configuration differs from theirs in three respects. 1) Our waveguide has magnetic side walls rather than metal side walls. This changes the modes of the guide. 2) Ours is a slot configuration rather than a post configuration; hence, we must apply Babinet's principle and duality. 3) Our problem is complicated by the presence of the dielectric substrate. The calculations are similar to those of Eisenhart and Khan, and it is unnecessary to give them here. We shall use the more common definition of admittance given by Harrington [10]. It is assumed that, in the slot, only the $y$-component of the electric field exists. We, therefore, excite only modes that are TE to the $x$ axis and, by symmetry, only those that have even symmetry about the $x$ and $y$ axes. The $y$-component of the electric field of the mode with indexes $m$, $n$ is of the form

$$
E_{y} \propto \cos \frac{2 m \pi x}{a} \cos \frac{2 n \pi y}{a} .
$$

Note that, unlike the case of a hollow metal waveguide, solutions exist for all nonnegative integers $m$ and $n$. The $H_{x}$ component is given by

$$
H_{x}=-Y_{m n} E_{y}
$$

where $Y_{m n}$ is the admittance of the $m, n$th mode given by Harrington: 


$$
Y_{m n}=\frac{k^{2}-(2 m \pi / a)^{2}}{\omega \mu \sqrt{k^{2}-(2 m \pi / a)^{2}-(2 n \pi / a)^{2}}} .
$$

Here $k$ is the propagation constant of plane waves in the material. We find that the susceptance of the gap is given by

$$
j B_{c}=2 Y_{0}^{-1}\left(1-\frac{g}{a}\right) \sum_{n=1}^{\infty}\left(\frac{\sin n \pi g / a}{n \pi g / a}\right)^{2}\left(Y_{o n}^{+}+Y_{o n}^{-}\right)
$$

where the $Y_{o n}^{+}$are the admittances looking in the $+z$ direction, the $Y_{o n}^{-}$are the admittances looking in the $-z$ direction, and $Y_{0}=\sqrt{\epsilon_{0} / \mu_{0}}$ is the characteristic admittance of free space. The factor $(1-g / a)$ is a phenomenological quantity that Eisenhart and Khan found necessary to insert in order for their theory to agree with experimental results. The lead susceptance is given by

$$
\left(j B_{l}\right)^{-1}=2 Y_{0} \sum_{m=1}^{\infty}\left[\frac{\left(\frac{\sin \frac{m \pi w}{a}}{\frac{m \pi w}{a}}\right)^{2}}{\sum_{n=0}^{\infty} \epsilon_{n}\left(\frac{\sin \frac{n \pi g}{a}}{\frac{n \pi g}{a}}\right)^{2}\left(Y_{m n}^{+}+Y_{m n}^{-}\right)}\right]
$$

where $\epsilon_{n}$ is the Neumann factor

$$
\epsilon_{n}= \begin{cases}\epsilon_{n}=1 & \text { if } n=0 \\ \epsilon_{n}=2 & \text { otherwise }\end{cases}
$$

These formulas will be used later to compare experimental results and theory and to predict the frequency response of the array.

\section{Measuring the Detectivity}

Measurements of detectivity were made using several different devices. However, the measurements reported in this section are all from the same device, which had a measured dc resistance of $74 \Omega$. The measurements are in three parts: a noise measurement, a VHF measurement at $140 \mathrm{MHz}$, and a $G$-band measurement at $215 \mathrm{GHz}$.

The noise of the array with a bias of $0.9 \mathrm{~V}$ was measured with a PAR 124 lock-in amplifier with type 118 preamp, in the frequency range of $10 \mathrm{~Hz}-100 \mathrm{kHz}$ (Fig. 7). In this range the largest component is $1 / f$ noise. The noise was calculated from $100 \mathrm{kHz}$ to $30 \mathrm{MHz}$ by assuming a combination of the same $1 / f$ noise and Johnson noise. The noise of the unbiased array was measured at $1 \mathrm{kHz}$ with a type 116 preamp. It was close to the calculated Johnson noise of the array, as expected.

The purpose of the VHF measurements was to determine the responsivity of the array of microbolometers independently of the coupling characteristics at $G$-band. They also compensate for the fact that the $G$-band klystron could only be modulated up to $10 \mathrm{kHz}$. The measurement setup for modulation frequencies between $30 \mathrm{~Hz}$ and $100 \mathrm{kHz}$ is shown in Fig. 8 . To obtain higher modulation frequencies, the outputs of two VHF signal generators at different frequencies were combined. In addition, the notch filter was replaced by a Lark low-pass

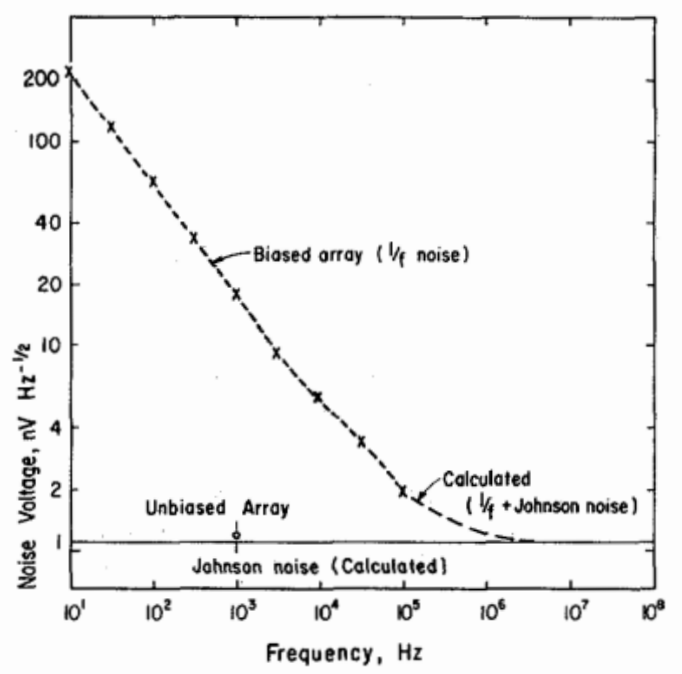

Fig. 7. Noise of microbolometer array with bias of $0.9 \mathrm{~V}$.

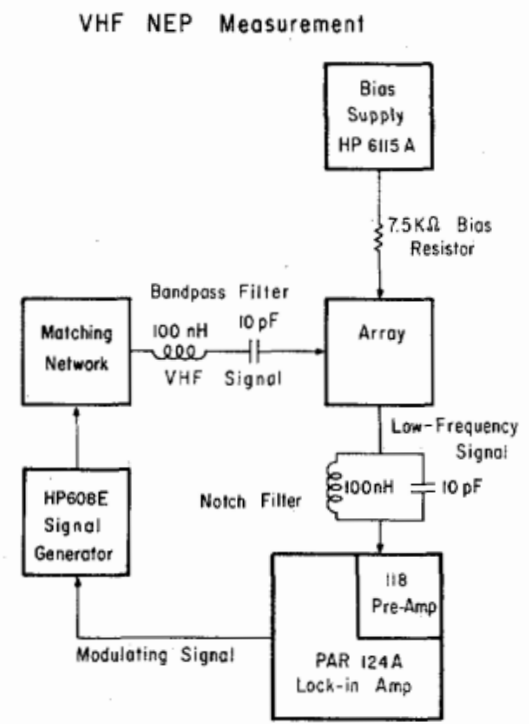

Fig. 8. Apparatus for VHF responsivity measurement.

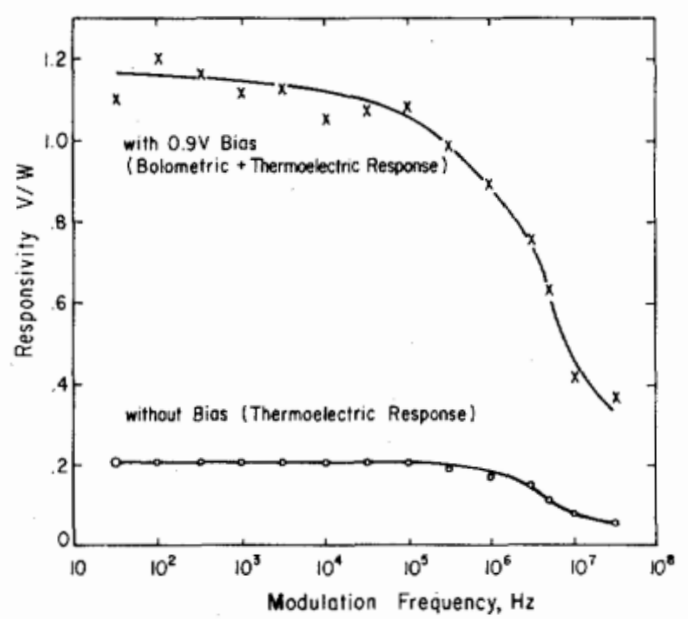

Fig. 9. Responsivity of microbolometer array, measured at $140 \mathrm{MHz}$.

filter with a cutoff at $75 \mathrm{MHz}$. Fig. 9 shows the measured responsivities with and without bias. The signal without bias $(0.2 \mathrm{~V} / \mathrm{W}$ at low frequencies) is of a thermoelectric nature, and appears to result from a slight, unintentional mask mis- 


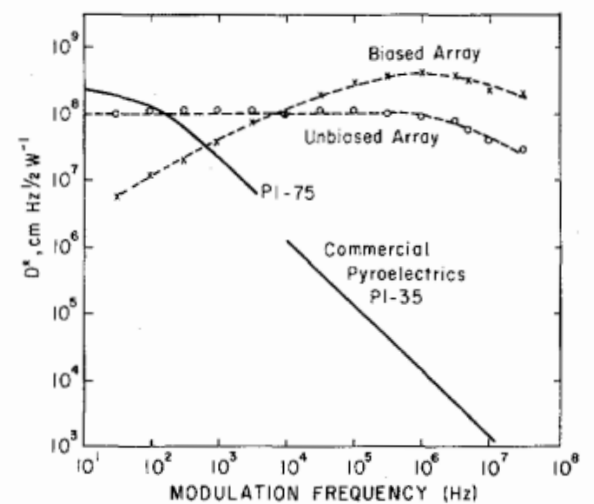

Fig. 10. Specific detectivities of biased and unbiased microbolometer arrays versus modulation frequency. Data for commercial pyroelectric detectors are shown for comparison.

alignment which makes one end of the bolometers more resistive than the other, causing a temperature gradient in the bismuth. The thermoelectric signals varied greatly from device to device and can probably be increased through appropriate redesign. The signal with bias ( $1.1 \mathrm{~V} / \mathrm{W}$ at low frequencies) is a combination of the bolometric signal and the thermoelectric signal. The $3 \mathrm{~dB}$ bandwidth of both signals was $5 \mathrm{MHz}$. From (13) we calculate the responsivity of a single microbolometer to be 20 times higher than these values, i.e., $4 \mathrm{~V} / \mathrm{W}$ without bias and $22 \mathrm{~V} / \mathrm{W}$ with bias.

Measurements at $G$-band were made using a Varian klystron with an output power of about $20 \mathrm{~mW}$. On the basis of comparison measurements with different calorimeters, we estimate the accuracy of the power measurements to be \pm 25 percent. The klystron excited a Microlab-FXR standard gain horn. The power incident on the array was calculated from the gain of the horn $(25.7 \mathrm{~dB}$ from standard-gain curves [12]) and the distance from the horn to the array $(37.5 \mathrm{~cm})$. The copper tuning short was aligned parallel to the quartz substrate with an $\mathrm{He}-\mathrm{Ne}$ laser. The coupling efficiency of the array was then determined by comparing the responsitivity at $215 \mathrm{GHz}$ with the previously measured responsivity at $140 \mathrm{MHz}$. This coupling efficiency was typically in the range $40-60$ percent. For the array whose characteristics are shown in Figs. 7 and 9, the coupling efficiency was 60 percent \pm 20 percent.

The value of $D^{*}$ was calculated from (15) and (16) using the results of Figs. 8 and 9 and multiplying by 0.6 , the experimentally determined coupling efficiency. The results are plotted as a function of modulation frequency in Fig. 10. For comparison, the detectivities of two large commercial pyroelectric detectors, from data taken by the manufacturer at much shorter wavelengths, are also shown in the figure. The detectivity of pyroelectrics at millimeter wavelengths is not known, but there are indications that values four times smaller than those shown may be appropriate [11]. We see that the multimode antenna has a higher detectivity than the pyroelectric detector at all modulation frequencies above $100 \mathrm{~Hz}$. The unbiased array gives the largest $D^{*}\left(10^{8} \mathrm{~cm} \cdot \mathrm{Hz}^{1 / 2} / \mathrm{W}\right)$ between $100 \mathrm{~Hz}$ and $10 \mathrm{kHz}$, while the biased array gives the largest $D^{*}$ at higher frequencies with a maximum of $4 \times 10^{8}$ at $1 \mathrm{MHz}$, more than four orders of magnitude larger than the detectivity of the pyroelectric detector at this modulation frequency.

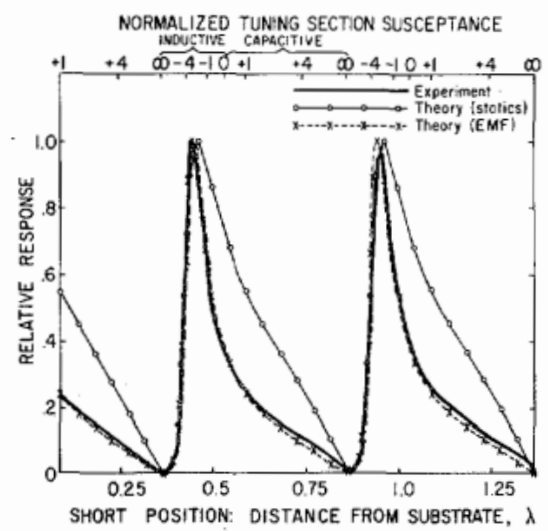

Fig. 11. Responsivity versus position of tuning short. Theoretical curves based on quasi-static and EMF theories are also shown.

\section{TUNing THE Array}

A further series of measurements was made in order to observe the effect of the adjustable tuning short, and to determine the dependence of responsivity on the angle of incidence of the beam. The array used in these measurements (not the same one used in the experiments of the preceding section) had a resistance of $88 \Omega$, and its coupling efficiency, measured by the methods described above, was 50 percent. Fig. 11 shows the received power as a function of the distance of the short from the face of the substrate nearest to it. From transmission-line theory we know that the period of this curve is $\lambda / 2$; thus, we can calculate the klystron's frequency. We can also determine the position of the short because we know that a zero of received power indicates that the impedance seen by the array, looking first into the substrate, then the air space, and finally the short, is zero. Using the known thickness of the substrate $(126 \mu \mathrm{m})$ we obtain the position scale shown at the bottom of the figure. The susceptance of the short, referred to the position of the array at the front surface, can then be calculated from transmission-line theory; it is shown at the top of the figure. The tuning curves are interesting because they are quite sensitive to the circuit parameters of the array that can be calculated from theory. Using the quasistatic formulas (17) and (18), the capacitive susceptance $B_{c}$ of Fig. 5(a) is calculated to be 4.60 (normalized to free space), and the inductive susceptance $B_{l}$ (normalized to free space) is calculated to be -4.17 . With these circuit values and the measured dc resistance of the array $(88 \Omega)$, a theoretical tuning curve was calculated and plotted in Fig. 11. The curve does not agree well with the measured tuning curve. We next calculated the circuit values by the EMF method using (21), (22), and (23). Terms in $n$ were included up to $n=a / g$ (5 terms) and in $m$ up to $m=a / w$ ( 83 terms). Eisenhart and Kahn show that the truncation error with these limits is about 1 percent. The dielectric substrate complicated this calculation. Different wave admittances had to be calculated for the waves propagated into the air and down into the substrate. The wave admittances down into the substrate had to be calculated by transmission-line methods, considering the effect of the air behind the substrate and taking the thickness of the substrate into account. The result is $B_{c}=4.67$ and $B_{l}=-2.63$. The circuit parameters for the simplified circuit [Fig. 5(b)] are 
(a)

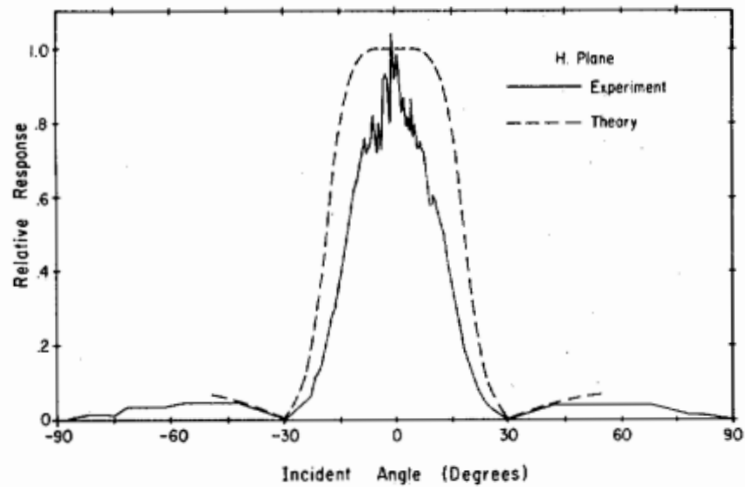

(b)

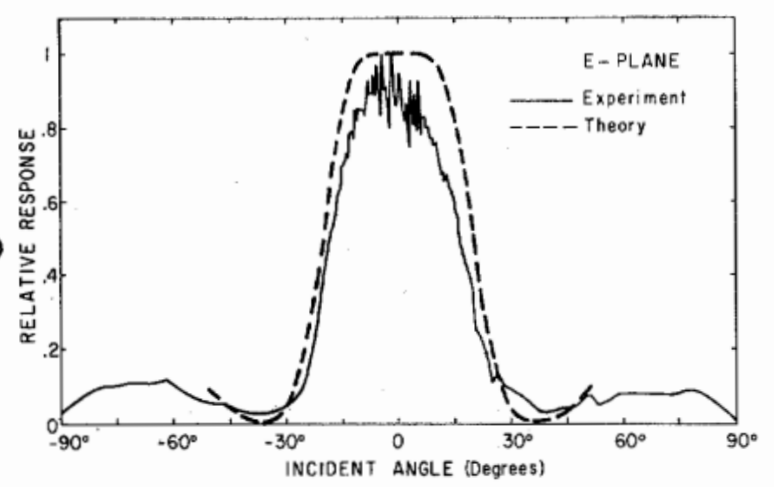

Fig. 12. (a) $H$-plane pattern of array. Solid curve, experimental; dashed curve, theoretical. (b) E-plane pattern of array. Solid curve, experimental; dashed curve, theoretical.

then $B=2.77$ and $G=1.17$. The tuning curve calculated from these parameters is also plotted in Fig. 11; it gives much better agreement with the experimental results.

When this array is tuned for maximum response, its normalized admittance is calculated to be 1.17. This implies a good match to free space and 99 percent of the incident power ought to be absorbed, in apparent contradiction to the measured coupling efficiency of 50 percent. To resolve this discrepancy, it would be helpful to have more accurate power measurements, as well as measurements of the reflected wave generated by the antenna and the losses of the horn.

The $H$ - and $E$-plane patterns are plotted in Fig. 12(a) and (b). These patterns show considerable fine structure near the peaks, probably a consequence of the fact that the array has only 20 rows and columns. No theory is available to predict how the circuit parameters of Fig. 5(b) will change as a function of angle. A crude estimation of the pattern can be made by assuming that $B_{c}$ and $B_{l}$ remain constant, so that the pattern is mainly due to changes in the wave impedance of the incident wave and the tuning section. The patterns calculated with this theory are shown in Fig. 12(a) and (b). There is some agreement, but clearly a more accurate theory is called for.

Because the source being used was not tunable, it was not possible to measure the response of the array as a function of frequency. However, the frequency response has been calculated by means of the EMF theory, with the results shown in Fig. 13. The dashed curve shows the frequency response when the array is fixed-tuned for maximum response at $215 \mathrm{GHz}$. The bandwidth is seen to be about 10 percent. The solid curve shows the response when the array is retuned for opti-

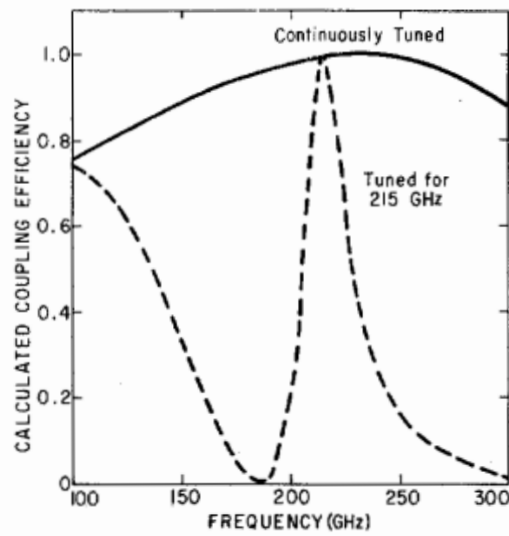

Fig. 13. Frequency response of array. Dashed curve: with fixed tuning; solid curve: with tuning optimized for each frequency.

mum response at each frequency. This indicated that a single array can be used over the entire range $100-300 \mathrm{GHz}$. Above $300 \mathrm{GHz}$, however, the array will begin to act as a diffraction grating, and the response will probably be reduced.

\section{CONCLUSIONS}

Multimode arrays provide a way to generate detectors with large area, using small circuit elements to perform the actual detection. Our experimental version, using microbolometers as detector elements, has maximum detectivity comparable to that of pyroelectrics, as well as much greater bandwidth. Detectors of this type should be useful in various applications requiring room-temperature detection of radiation with wavelengths down to perhaps $50 \mu \mathrm{m}$. Replacement of the microbolometers with Schottky diodes or SIS or MBM junctions should lead to great improvements in speed and detectivity. On the basis of the agreement between tuning experiments and calculations, we believe the EMF method to be a reasonably accurate theory for design of the device.

\section{ACKNOWLEDGMENT}

We wish to thank T. Van Duzer, D. J. Angelakos, and K. K. Mei for their suggestions; D. T. Hodges, J. R. Tucker, and E. J. Danielewicz of the Aerospace Corporation for their hospitality and the use of the klystron; E. E. Reber and H. Dyson for their expert assistance in the measurements; and the reviewers for their helpful comments.

\section{REFERENCES}

[1] C. Kittel, Thermal Physics. New York: Wiley, 1969, p. 403.

[2] F. R. Arams, Infrared-to-Millimeter Wavelength Detectors. Dedham, MA: Artech, 1973, p. 11.

[3] R. E. Collin and F. J. Zucker, Antenna Theory Part 1. New York: McGraw-Hill, 1969, p. 117.

[4] T. L. Hwang, S. E. Schwarz, and D. B. Rutledge, "Microbolometers for infrared detection," Appl. Phys. Lett., vol. 34, pp. 773776, June, 1979.

[5] B. J. Clifton, R. A. Murphy, and G. D. Alley, "Integrated monolithic mixers on GaAs for millimeter and submillimeter wave applications," in Conf. Dig. 4th Int. Conf. Infrared and Millimeter Waves and Their Applications, IEEE Cat. 79CH1384-7MTT, Dec. 1979 , pp. 84-86.

[6] P. L. Richards, T. M. Shen, R. E. Harris, and F. L. Lloyd, "Superconductor-insulator-superconductor quasiparticle junctions as microwave photon detectors," Appl. Phys. Lett., vol. 36, pp. 480-482, Mar. 15, 1980. 
[7] R. H. Kingston, Detection of Optical and Infrared Radiation. New York: Springer-Verlag, 1978, p. 22.

[8] R. L. Eisenhart and P. J. Khan, "Theoretical and experimental analysis of a waveguide mounting structure," IEEE Trans. Microwave Theory Tech., vol. MTT-19, pp. 706-719, Aug. 1971.

[9] R.E. Collin, Field Theory of Guided Waves. New York: McGrawHill, 1960.

[10] R. F. Harrington, Time-Harmonic Electromagnetic Fields. New York: McGraw-Hill, 1961, pp. 152-155.

[11] F. B. Foote and D. T. Hodges, "Calibration of power and energy meters for the far infrared/near-millimeter wave spectral region," in Conf. Dig. 4th Int. Conf Infrared and Millimeter Waves and Their Applications, IEEE Cat. 79CH1384-7MTT, Dec. 1979, pp. 79-88.

[12] H. Jasik, Antenna Engineering Handbook. New York: McGrawHill, 1961, pp. 7-10.

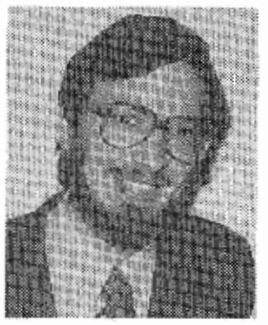

D. B. Rutledge (S'75-M'80) was born in Savannah, GA, on January 12,1952 . He received the B.A. degree in mathematics from Williams College, Williamstown, MA, in 1973, the M.A. degree in electrical engineering from Cambridge University, Cambridge, England, in 1975, and the $\mathrm{Ph} . \mathrm{D}$. degree in electrical engineering from the University of California, Berkeley.

From 1975 to 1976 he was associated with the communications group of the General Dynamics Corporation, where he worked with airborne microwave data systems. In 1980 he was appointed an Assistant Professor of Electrical Engineering at the California Institute of Technology, Pasadena. He is working on millimeter and submillimeter devices and their applications.

Mr. Rutledge is a member of Phi Beta Kappa and the American Physical Society.

S. E. Schwarz was born in Los Angeles, CA, on January 29, 1939. He received the B.S. degree in physics from the California Institute of Technology, Pasadena, in 1959, the A.M. degree in physics from Harvard University, Cambridge, MA, in 1961, and the Ph.D. degree in electrical engineering from California Institute of Technology, in 1964.

He has held positions with Hughes Research Laboratories, Bell Laboratories, and IBM Research Laboratories. Since 1964 he has been a faculty member of the Department of Electrical Engineering and Computer Sciences at the University of California, Berkeley, where he is engaged in work on millimeter-wave and infrared devices and quantum electronics.

Prof. Schwarz held a Guggenheim Fellowship in 1971-1972.

\title{
$\mathrm{He}-\mathrm{Cd}^{+}$White Light Laser by a Novel Tube Structure
}

\author{
MASAHIRO OTAKA, TAKASHI OSHIMA, MANABU TAKEUCHI, TOSHIO OIKAWA, AND K. FUJII
}

\begin{abstract}
A newly designed external cathode type $\mathrm{He}-\mathrm{Cd}^{+}$hollowcathode white light laser has been realized by using a "twin cathode" and a cylindrical grid electrode which is distributed coaxially along the rod anode. The electric input power of this system is $300 \mathrm{~V}, 1 \mathrm{~A}$, and $300 \mathrm{~W}$, almost double that of the flute type, $250 \mathrm{~V}, 0.6 \mathrm{~A}$, and $150 \mathrm{~W}$, but the cathode temperature is almost the same in both systems. The output power of the blue laser line was increased over that of the flute and hybrid hole types, while that of the green and red laser lines could not exceed both types.
\end{abstract}

$\mathrm{A}^{\mathrm{s}}$ the $\mathrm{He}-\mathrm{Cd}^{+}$hollow-cathode white light laser, consisting of red, green, and blue laser beams, shows excellent color regeneration characteristics, we have continued our studies toward an improvement in this system. The flute type [1], which was developed first, has good operational characteristics, for example, low threshold current for laser oscillation, a properly balanced output power ratio of the three colors, stable

Manuscript received April 2, 1980; revised October 6, 1980.

M. Otaka, M. Takeuchi, T. Oikawa, and K. Fujii are with the Department of Electrical Engineering, Ibaraki University, Hitachi-shi, Ibaraki, Japan.

T. Oshima is with the Yokosuka Electrical Communication Laboratory, Yokosuka-shi, Kanawaga, Japan.

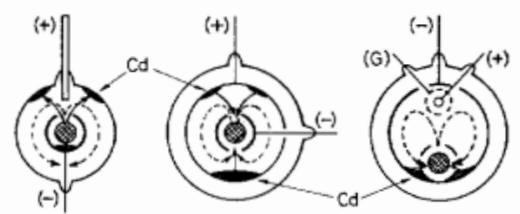

(a)

(b)

(c)

Fig. 1. End view of laser tubes for white light laser oscillation: (a) flute type, (b) concentric cylinder type, (c) twin-cathode type, where Cd is the cadmium metal, $G$ is the grid electrode, the meshed circle is the lasing zone, the solid arrow shows the diffusion of $\mathrm{Cd}$ vapor, and the dotted arrow shows the convection of buffer gas (He).

running by series resistance connected with each of the multiple anodes, and so on, but its laser operational lifetime is basically short since the $\mathrm{Cd}$ metal is lost by the deposition of the Cd vapor onto the cold glass envelope [see Fig. 1(a)].

Concentric cylinder types [2]-[4], which use a large diameter tube as the anode and a smaller one as the cathode, were used in order to increase the laser operational lifetime because not only can a lot of $\mathrm{Cd}$ metal be stored inside the large cylindrical anode, but also the loss of the $\mathrm{Cd}$ metal by the deposition is less than the flute type [see Fig. 1(b)]. Fortunately, the structure of this system is simple, convenient to manufac- 